\title{
GENERATING IDEALS UP TO PROJECTIVE EQUIVALENCE
}

\author{
D. KATZ
}

(Communicated by Eric Friedlander)

\begin{abstract}
It is shown that every ideal in a commutative Noetherian ring of dimension $d$ is projectively equivalent to an ideal having $d+1$ generators.
\end{abstract}

\section{INTRODUCTION}

Let $R$ be a $d$-dimensional Noetherian ring and $I \subseteq R$ an ideal. A wellknown theorem, whose origin dates back to Kronecker, asserts that there exists a $(d+1)$-generated ideal $J \subseteq R$ such that $I$ and $J$ have the same radical. In other words, $I$ is generated up to radical by $d+1$ elements. An important improvement was given in [2], where it was shown that if $R=A[X]$ is a polynomial ring then $I$ can be generated up to radical by $d$ elements. This has the fundamental consequence that every algebraic set in affine $d$-space can be cut out by $d$ hypersurfaces. In [1] Boratynski gave a common antecedent for these two theorems by showing that there exists an ideal $J$ with the same radical as $I$ such that $\mu\left(J / J^{2}\right) \leq d$ (where $\mu($ ) denotes a minimal number of generators).

In this note we offer an improvement of these results by demonstrating the following theorem.

Theorem. Let $R$ be a d-dimensional Noetherian ring and $I \subseteq R$ any ideal. Then there exists an ideal $J$ projectively equivalent to I satisfying $\mu\left(J / J^{2}\right) \leq d$.

The notion of projective equivalence is defined below, but for now it suffices to say that it is a substantially stronger condition than having the same radical. A consequence of this theorem is that one obtains a more direct path to the following important geometric result (proven in [3]). The defining ideal for any nonsingular variety in affine $d$-space can be generated by $d$ or fewer elements.

Throughout, $R$ will be a commutative Noetherian ring. Recall that an ideal $J \subseteq I$ is a reduction of $I$ if $J I^{p}=I^{p+1}$ for some $p \geq 1$. Given an ideal $I$, there is a unique largest ideal reduced by $I$ called the integral closure of $I$.

Received by the editors May 4, 1992; presented at the special session in Commutative Algebra at the 873rd meeting of the AMS, Springfield, Missouri, March 20, 1992.

1991 Mathematics Subject Classification. Primary 13A15.

Key words and phrases. Projectively equivalent ideals. 
Ideals $I$ and $J$ are said to be projectively equivalent if some power of $I$ and some power (usually different) of $J$ have the same integral closure. In particular, projectively equivalent ideals have the same radical. McAdam's text [7] is a good reference for reductions, integral closure, and projective equivalence.

The basic idea of our proof is relatively straightforward. We observe that if the ideal $I$ is contained in the Jacobson radical, then the usual results about reductions in local rings still apply (see [8]). We then localize at the multiplicatively closed set which "radicalizes $I$ " and increase the estimate from the local case by one. We begin with the following superficial element lemma. Typically the statement and proof of this lemma are given in the context of local or semilocal rings. However, essentially the same proof works in the nonlocal case, so we have only sketched the argument below.

Superficial element lemma. Let $I \subseteq R$ be a regular ideal, i.e., $(0: I)=0$. Then:

(i) there exists an $n \geq 1$ and a nonzero divisor $x \in I^{n}$ satisfying ( $I^{n t}$ : $x)=\left(I^{n}\right)^{t-1}, t \gg 0$.

(ii) For $n \geq 1$ and $x \in I^{n}$ in (i), $\left(I^{n k t}: x^{k}\right)=\left(I^{n k}\right)^{t-1}, t \gg 0$, for all $k \geq 1$.

(iii) If $R$ has infinite residue fields, we may take $n=1$ in (i).

Sketch of proof. Write $\mathscr{R}=\bigoplus_{m \geq 0} I^{m} t^{m} \subseteq R[t], t$ an indeterminate, for the Rees ring of $R$ with respect to $I$, and let $\left\{\mathscr{P}_{1}, \ldots, \mathscr{P}_{r}\right\}$ be the union of Ass $\mathscr{R}$ and the relevant prime divisors of $I \mathscr{R}$. To prove (i), we may employ the homogeneous prime avoidance lemma to select an element $x t^{n}$ of $\mathscr{R}$ not contained in any $\mathscr{P}_{i}$. This $n$ and $x$ work. The proof of (ii) is easy. To prove (iii), let $J_{i}=\left\{x \in R \mid x t \in \mathscr{P}_{i}\right\}$. Then $J_{i}$ is properly contained in $I$. If $R$ has infinite residue fields, then by McAdam's prime avoidance lemma (see [6]) we may select $x$ belonging to $I$, not in any $J_{i}$; this $x$ works.

Proposition. Let $R$ be a d-dimensional Noetherian ring and $I$ an ideal contained in the Jacobson radical. Then:

(i) some power of I has a $d$-generated reduction,

(ii) if $R$ has infinite residue fields, I has a $d$-generated reduction.

Proof. Proceed by induction on $d$. If $d=0$, then $I$ is contained in the nilradical of $R$, and the result is obvious. Suppose $d \geq 1$ and $(0: I)=0$. Select $n \geq 1$ and $x \in I^{n}$ according to the first part of the lemma. By induction, there exists $k \geq 1$ and a $(d-1)$-generated ideal $J \subseteq I^{n k}$ such that $J$ reduces $I^{n k}$ modulo $x R$. Thus $J$ reduces $I^{n k}$ modulo $x^{k} R$. (Recall that, for ideals $A, B$, and $C, A$ reduces $B$ modulo $C$ if and only if $A$ reduces $B$ modulo the radical of $C$.) Thus for $t \gg 0$

$$
\left(I^{n k}\right)^{t+1} \subseteq J\left(I^{n k}\right)^{t}+x^{k} R
$$

therefore,

$$
\left(I^{n k}\right)^{t+1}=J\left(I^{n k}\right)^{t}+\left(\left(I^{n k}\right)^{t+1}: x^{k}\right) x^{k}=J\left(I^{n k t}\right)+\left(I^{n k}\right)^{t} x^{k},
$$

by Lemma (ii). Hence $\left(J, x^{k}\right)$ reduces $I^{n k}$. If $(0: I) \neq 0$, let $L=\left(0: I^{p}\right)$, $p \gg 0$. From the preceding case, we may find a power $m$ of $I$ and a $d$ generated reduction $J \subseteq I^{m}$ such that $J$ reduces $I^{m}$ modulo $L$. Thus, for $t \gg 0,\left(I^{m}\right)^{t+1} \subseteq J I^{m t}+L$. If we multiply by $I^{p m}$, we see that $J$ reduces $I^{m}$. 
Finally, if $R$ has infinite residue fields, we may repeat the argument, always taking superficial elements from the first power of $I$.

Theorem. Let $R$ be a Noetherian ring and $I \subseteq R$ an ideal. Let $d$ be the maximum of the heights of maximal ideals containing $I$, and suppose $d<\infty$. Then:

(i) Some power of I admits a reduction $J$ satisfying $\mu\left(J / J^{2}\right) \leq d$.

(ii) If $R$ has infinite residue fields, then $I$ admits a reduction $J$ satisfying $\mu\left(J / J^{2}\right) \leq d$.

Proof. Set $S=1+I$, and localize $R$ at $S$. By the proposition, there exists $n \geq 1$ and a $d$-generated ideal $K \subseteq I_{S}^{n}$ such that $K$ is a reduction of $I_{S}^{n}$. Thus for $t \gg 0$

$$
K\left(I_{S}^{n t}\right)=I_{S}^{n t+n} .
$$

Let $J$ be the complete inverse image of $K$. Then:

(a) $s J \subseteq I^{n}$ for some $s=1-x \in S$. Multiplying by $1+x+x^{2}+\cdots+x^{n-1}$, we obtain $\left(1-x^{n}\right) J \subseteq I^{n}$, so $J \subseteq I^{n}$.

(b) $s\left(I^{n}\right)^{t+1} \subseteq J I^{n t} \subseteq J$ for some $s \in S$. This implies $\left(I^{n}\right)^{t+1} \subseteq J$ (by definition of $J$ ). Hence $I$ and $J$ have the same radical. It now follows from $(*)$ that $J$ reduces $I^{n}$.

(c) Since $\operatorname{rad}(I)=\operatorname{rad}(J)$, it follows that $R_{S}=R_{T}$ for $T=1+J$. Hence $\mu\left(J_{T}\right) \leq d$, so $\mu\left(J / J^{2}\right) \leq d$.

If $R$ has infinite residue fields, the proof is similar and uses the second part of the proposition.

Corollary A. Let $R, I$, and $d$ be as in the theorem. Then:

(i) Some power of I has a $(d+1)$-generated reduction.

(ii) If $R$ has infinite residue fields, then I has a $(d+1)$-generated reduction.

(iii) If $R=A[X]$ is a polynomial ring and $d=\operatorname{dim} R$, we may take $d$ in place of $d+1$ in (i) and (ii).

In particular, any ideal in a d-dimensional Noetherian ring $R$ is projectively equivalent to a $(d+1)$-generated ideal. If $R=A[X]$ is a polynomial ring, we may take $d$ in place of $d+1$.

Proof. (i) and (ii) follow from the theorem since $\mu\left(I / I^{2}\right) \leq d$ implies $\mu(I) \leq$ $d+1$. For (iii), we must use the following result of Mohan Kumar. If $R=A[X]$ is a polynomial ring and $\mu\left(J / J^{2}\right)=d$, where $d=\operatorname{dim} R$, then $\mu\left(J / J^{2}\right)=$ $\mu(J)$. This result is proved, though not stated explicitly in [3] (see the proof of Theorem 2 there). Actually, Mohan Kumar assumes that $R$ is reduced and $\operatorname{height}(J)>0$, but this is sufficient for our purposes. Indeed, suppose $J \subseteq I^{n}$ is a reduction and $\mu\left(J / J^{2}\right) \leq d$. If $\mu\left(J / J^{2}\right)<d$, then $\mu(J) \leq d$. Suppose $\mu\left(J / J^{2}\right)=d$. If $J$ is nilpotent, $I$ is nilpotent, so $I^{n}$ has a 0 -generated reduction. If $J$ is not nilpotent, let $L$ be the intersection of the height zero primes not containing $J$. Suppose we could show $\mu(J+L / L) \leq d$. Then there exists an ideal $K \subseteq J$ with $\mu(K) \leq d$ and $K+L=J+L$. Thus $J=K+(L \cap J)$. But $L \cap J$ is nilpotent, so we have that $K$ reduces $I^{n}$ and $\mu(K) \leq d$.

To see $\mu(J+L / L) \leq d$, observe that $\mu\left(J / J^{2}\right) \leq d$ implies $\mu\left(J+L / J^{2}+L\right) \leq$ $d$. If $\mu\left(J+L / J^{2}+L\right)<d, \mu(J+L / L) \leq d$. If $\mu\left(J+L / J^{2}+L\right)=d$ and 
$d>\operatorname{dim}(R / L)$, then $d=\mu\left(J+L / J^{2}+L\right)=\mu(J+L / L)$ (see the remark below). Finally, if $\mu\left(J+L / J^{2}+L\right)=d$ and $d=\operatorname{dim}(R / L)$, we can apply Mohan Kumar's result to conclude $\mu(J+L / L)=d$.

The next corollary shows that one may use reductions as a path to obtain certain Forester-Swan type results. In particular, the theorem above together with Mohan Kumar's result yield a quick path to the following important theorem (see [3]): Let $P \subseteq k\left[X_{1}, \ldots, X_{d}\right]$ define a nonsingular variety in affine $d$-space over the field $k$. Then $\mu(P) \leq d$.

Corollary $\mathbf{B}$ (see $[3,4])$. Let $R$ be a d-dimensional Noetherian ring with infinite residue fields. Suppose that $I \subseteq R$ is a local complete intersection (i.e., $\mu\left(I_{M}\right)=$ height $I_{M}$ for all maximal ideals containing $\left.I\right)$. Then:

(i) $\mu(I) \leq d+1$.

(ii) If $R=A[X]$ is a polynomial ring, $\mu(I) \leq d$.

Proof. Let $J \subseteq I$ be a reduction satisfying $\mu\left(J / J^{2}\right) \leq d$. Then $J_{M}=I_{M}$ for all maximal ideals containing $I$ (and hence $J$ ). Thus $J=I$, and the result follows along the same lines as the previous corollary.

Remark. (i) Mohan Kumar's result mentioned in Corollary A is technically the most difficult part of his solution to the Eisenbud-Evans conjectures, in that it uses a number of deep-patching arguments along the lines of Quillen's patching theorem. It represents an improvement by one in the well-known (and considerably easier to prove) fact: If an ideal $I$ in a $d$-dimensional Noetherian ring satisfies $\mu\left(I / I^{2}\right)=d+1$, then $\mu(I)=d+1$ (see [4]). If one could circumvent the patcking arguments, this would yield an even more direct route to Corollary B.

(ii) A special case of Corollary $\mathrm{A}$ (iii) for $R=k\left[X_{1}, \ldots, X_{d}\right]$ was given by Lyubesnik in [5]. Here, too, use is made of Mohan Kumar's result [3, Theorem 5].

\section{ACKNOWLEDGMENT}

Some of the work on this paper was done during the spring of 1991 while I was visiting Purdue University; I would like to thank the mathematics department, in particular, Bill Heinzer and Craig Huneke, for the hospitality I received during my stay.

\section{REFERENCES}

1. H. Boratynski, Generating ideals up to radical, Arch. Math. 33 (1979), 423-425.

2. D. Eisenbud and E. G. Evans, Jr., Every algebraic set in $n$-space is the intersection of $n$ hypersurfaces, Invent. Math. 19 (1973), 107-112.

3. N. Mohan Kumar, On two conjectures about polynomial rings, Invent. Math. 40 (1978), 225-236.

4. E. Kunz, Introduction to commutative algebra and algebraic geometry, Birkhäuser, Boston, MA, 1985.

5. G. Lyubesnik, A property of ideals in polynomial rings, Proc. Amer. Math. Soc. 98 (1986), 399-400.

6. S. McAdam, Finite covering by ideals, Ring Theory (Proceedings of the Oklahoma Conference), Lecture Notes in Pure and Appl. Math., vol. 7, Marcel Dekker, New York, 1974, pp. 163-171. 
7. __ Asymptotic prime divisor, Lectures Notes in Math., vol. 1023, Springer, Berlin, 1983.

8. D. G. Northcott and D. Rees, Reductions of ideals in local rings, Math. Proc. Cambridge Philos. Soc. 50 (1954), 145-158.

Department of Mathematics, University of Kansas, Lawrence, Kansas 66045

E-mail address: dlk@mercury .math.ukans . edu 